\title{
Hyperplasie surrénalienne par bloc en 21 hydroxylase : une cause rare d'hypofertilité masculine et de tumeur du testicule
}

\author{
Dominique DELAVIERRE ${ }^{1}$, Philippe EMY2, Marylise DOLLEY ${ }^{3}$, Bruno GUTHAUSER ${ }^{4}$
}

1 Urologie-Andrologie, CHR d'Orléans, 2 Endocrinologie, CHR d'Orléans, 3 Gynécologie-Obstétrique, CHG de Dreux, 4 Biologie de la reproduction, CHG de Dreux

\section{RÉSUMÉ}

Les auteurs rapportent un cas de tumeur testiculaire bilatérale correspondant à des inclusions surrénaliennes intra-testiculaires, découverte lors d'un bilan d'hypofertilité chez un homme de 29 ans traité pour une hyperplasie congénitale des surrénales par bloc en 21 hydroxylase.

Liées à une stimulation chronique par l'ACTH les inclusions surrénaliennes intra-testiculaires des blocs en 21 hydroxylase témoignent souvent d'une réponse insuffisante au traitement freinateur par les glucocorticoïdes. L'hypofertilité est liée à l'effet de masse des nodules tumoraux, aux conséquences toxiques des stéroïdes surrénaliens et à un hypogonadisme hypogonadotrophique.

Chez un homme présentant une hyperplasie des surrénales par bloc en 21 hydroxylase et une hypofertilité il est indispensable de rechercher des inclusions surrénaliennes intra-testiculaires. De même il est important de rechercher à l'interrogatoire des antécédents de bloc en 21 hydroxylase face à des nodules testiculaires bilatéraux.

Mots-clés : tumeur du testicule, hyperplasie des surrénales, bloc en 21 hydroxylase, hypofertilité masculine

\section{OBSERVATION}

Un homme de 29 ans est adressé en consultation d'uroandrologie pour prise en charge d'une tumeur testiculaire bilatérale découverte sur une échographie lors d'un bilan d'hypofertilité (oligoasthénotératospermie sévère avec volume éjaculé normal). Cette échographie montre la présence de plusieurs nodules hétérogènes des deux testicules avec des épididymes sains (Figure 1). Le patient a des antécédents d'hyperplasie congénitale des surrénales par bloc en 21 hydroxylase et reçoit de l'hydrocortisone. Il est asymptomatique sur le plan uro-génito-sexuel et ne présente pas de signes d'hypogonadisme. L'examen palpe plusieurs nodules indurés des deux testicules, sans anomalie des épididymes et des déférents, et sans varicocèle. Le bilan hormonal (testostérone totale, LH, FSH, prolactine et $17 \beta$ estradiol) est normal. La $17 \alpha$ hydroxyprogestérone est à $100,2 \mathrm{ng} / \mathrm{ml}(\mathrm{N}<2,5 \mathrm{ng} / \mathrm{ml})$ et l'ACTH à 5,1 $\mathrm{ng} / \mathrm{ml}$ ( $N 9$ à 52). La spermoculture est stérile. Le caryotype est $46 \mathrm{XY}$. Les marqueurs tumoraux testiculaires sont négatifs.

\section{DISCUSSION}

Ce patient présente des inclusions surrénaliennes intratesticulaires en rapport avec sa maladie endocrinienne.

L'hyperplasie congénitale des surrénales par bloc en 21 hydroxylase est due à une stimulation corticosurrénalienne sous l'influence de l'ACTH hypophysaire. II existe en effet

Correspondance :

Dr Dominique DELAVIERRE - Service d'Uro-Andrologie, CHR La Source, BP 6709, 45067 Orléans Cedex 2 Tel 0238514626 - Fax 0238514159 - Email delav.uro@wanadoo.fr 

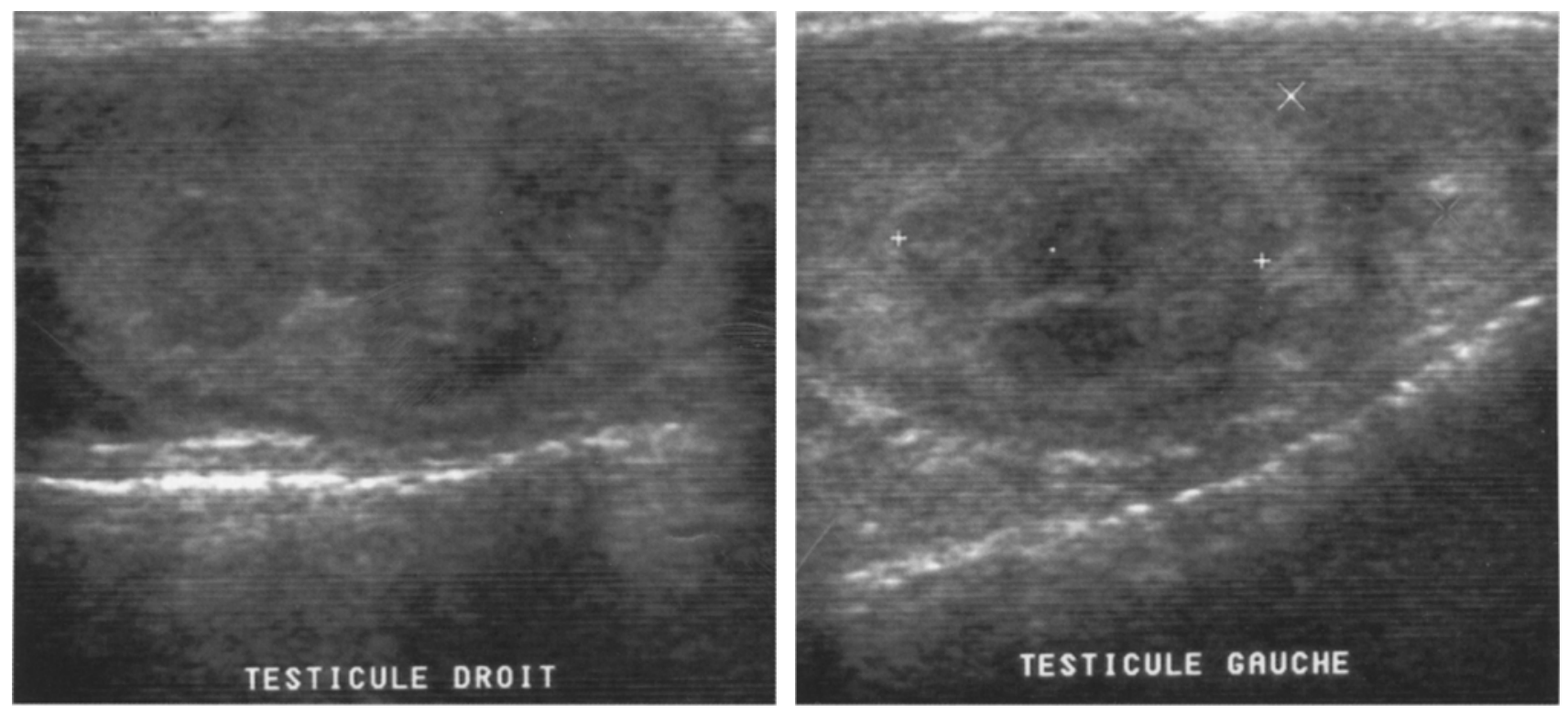

\section{Figure 1 : Echographie des testicules avec nodules multiples et bilatéraux.}

une défreination hypophysaire d'ACTH par manque de cortisol secondaire au blocage dans la chaine de synthèse. $\mathrm{Ce}$ blocage entraîne une accumulation de progestérone et de $17 \alpha$ hydroxyprogestérone.

Les inclusions surrénaliennes intra-testiculaires survenant lors d'hyperplasie des surrénales par bloc en 21 hydroxylase sont des tumeurs bénignes développées à partir de reliquats de tissu surrénalien intra-testiculaire physiologiquement présents chez les enfants [5,6]. Elles sont fréquentes, asymptomatiques, volontiers multiples et bilatérales.

Histologiquement proches des tumeurs à cellules de Leydig, elles ne contiennent pas de cristalloïdes de Reinke [4].

Liées à une stimulation chronique par l'ACTH, elles sont plus fréquentes dans les formes sévères de la maladie avec perte de sel à la naissance [5]. Elles témoignent souvent d'une réponse insuffisante au traitement freinateur par les glucocorticoïdes mais pas toujours, comme l'illustrent notre exemple et d'autres cas de la littérature [6]. La présence d'inclusions surrénaliennes malgré un traitement adapté trouverait son explication dans la génétique [6, 7]. En effet le bloc en 21 hydroxylase est du à des mutations du gène CYP21 codant pour cet enzyme. L'absence du gène CYP21 sur les 2 allèles entraînerait un défaut complet d'activité enzymatique avec des taux d'ACTH très élevés en période prénatale ce qui favoriserait le développement des inclusions surrénaliennes.

Lors d'hyperplasie des surrénales par bloc en 21 hydroxylase l'hypofertilité est secondaire à 3 mécanismes [1 - 6] :

- les nodules tumoraux réduisent et détruisent le parenchyme testiculaire sain et obstruent les canaux excréteurs (rete testis) ;

- les stéroïdes surrénaliens ont un effet délétère sur la spermatogenèse ;
- la sécrétion excessive d'androgènes surrénaliens entraîne un hypogonadisme hypogonadotrophique (rétrocontrôle négatif) majoré par l'élévation de la progestérone et de la $17 \alpha$ hydroxyprogestérone.

Chez un homme présentant une hyperplasie des surrénales par bloc en 21 hydroxylase et une hypofertilité, il est indispensable de rechercher des inclusions surrénaliennes testiculaires à l'examen clinique et à l'échographie car les nodules ne sont pas toujours palpables [1, 6]. En effet la découverte de ces inclusions peut conduire à intensifier le traitement, c'est à dire la freination de la sécrétion d'ACTH, afin d'en réduire le volume et d'améliorer la fonction exocrine des testicules. L'absence d'amélioration de la spermatogenèse justifie le recours aux techniques d'assistance médicale à la procréation. Une cryoconservation du sperme est également souhaitable afin de pallier à une dégradation éventuelle des paramètres spermatiques.

La méconnaissance de ce diagnostic pouvant conduire à une chirurgie inutile et mutilante dans l'hypothèse d'une tumeur maligne des testicules, il est également important de rechercher à l'interrogatoire des antécédents de bloc en 21 hydroxylase face à des nodules testiculaires bilatéraux.

\section{RÉFÉRENCES}

1. CABRERA M.S., VOGIATZI M.G., NEW M.I. : Long term outcome in adult males with classic congenital adrenal hyperplasia. J. Clin. Endocrinol. Metab., 2001, 86 : 3070-30788.

2. MIRSKY H.A., HINES J.H. : Infertility in a man with 21-hydroxylase deficient congenital adrenal hyperplasia. J. Urol., 1989, $142: 111-113$

3. MURPHY H., GEORGE C., DE KRETSER D., JUDD S. : Successful treatment with ICSI of infertility caused by azoospermia associated with adrenal rests in the testes: case report. Hum. Reprod., 2001, 16 : 263-267.

4. SAN MIGUEL FRAILE P., FERNANDEZ FERNANDEZ G., 
MEIJIDE RICO F. et al. : Hyperplasia of adrenal rests in the testicle : a rare cause of male infertility. Actas Urol. Esp., 2003, 27: 234-239.

5. SPIESS J., YOUNG J. : Anomalies de la reproduction chez les hommes nés avec un déficit sévère en 21 hydroxylase. Médecine clinique endocrinologie et diabète. $\mathrm{N}^{\circ}$ spécial "2ème journée d'endocrinologie sexuelle Alfred Jost : 6 février 2004" : 40-44.

6. STIKKELBROECK N.M., OTTEN B.J., PASIC A. et al. : High prevalence of testicular adrenal rest tumors, impaired spermatogenesis, and Leydig cell failure in adolescent and adult males with congenital adrenal hyperplasia. J. Clin. Endocrinol. Metab., 2001, $86:$ 5721-5728.

7. WHITE P.C., SPEISER P.W. : Congenital adrenal hyperplasia due to 21-hydroxylase deficiency. Endocr. Rev., 2000, 21 : 245291.

Manuscrit reçu : mars 2005 ; accepté avril 2005.

\begin{abstract}
Congenital adrenal hyperplasia due to 21-hydroxylase deficiency: A rare cause of male infertility and testicular tumor
\end{abstract}

\title{
Dominique DELAVIERRE, Philippe EMY, Marylise DOL- LEY, Bruno GUTHAUSER
}

The authors report the case of a 29-year-old man with bilateral testicular adrenal-like tumors in a context of congenital adrenal hyperplasia (CAH) due to 21-hydroxylase deficiency.

Inadequate suppression of ACTH secretion is a dominant etiological factor in the development of testicular masses in CAH.

Destruction of the testicular tubules by the adrenal rests and longstanding suppression of the pituitary-gonadal axis can lead to infertility.

The presence of testicular adrenal rests must be investigated in any man with CAH due to 21-hydroxylase deficiency and infertility. Similarly, it is important to investigate possible 21-hydroxylase deficiency in patients with bilateral testicular tumors.

Key-words: congenital adrenal hyperplasia, 21-hydroxylase deficiency, male infertility, testicular cancer 